\title{
Direct in situ GDD measurement in optical coating process
}

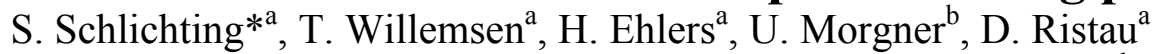 \\ ${ }^{a}$ Laser Zentrum Hannover e.V., Hollerithallee 8, 30419 Hanover, Germany; ${ }^{b}$ Institut für \\ Quantenoptik, Leibniz Universität Hannover, Welfengarten 1, 30167 Hanover, Germany
}

\begin{abstract}
In the presented work a fast frequency domain measurement system to determine group delay (GD) and group delay dispersion (GDD) of optical coatings is proposed. The measurements are performed in situ directly on moving substrates during the thin film coating process. The method is based on a Michelson interferometer, which is equipped with a high power broad band light source and a fast spectrometer. Especially for the production of chirped mirrors it is advantageous to obtain group delay and group delay dispersion data of the last layers. This additional information allows for online corrections of coating errors to enhance the precision of complex interference filters for short pulse applications.
\end{abstract}

Keywords: in situ measurement, GD, GDD, FTSI, STFT, optical coatings, optical thin films, chirped mirrors

\section{INTRODUCTION}

During the last years, short pulse lasers have gained increasing importance, for example in ultra-precise material processing. On the way to more power and shorter pulses the demands on the lasers increased rapidly. Today, key elements of many short pulse lasers are chirped mirrors for the compensation of dispersion effects induced within the beam path. To achieve these shorter pulses precise chirped mirrors with very flat broad negative group delay dispersion (GDD) or very high negative GDD are needed. This imposes critical demands on coating precision and coating control mechanisms. To deposit these complicated coating designs with more than hundred layers, advanced optical monitoring strategies like broad band monitoring and also single wavelength monitoring are state of the art. These techniques use transmittance or reflectance measurements during the coating process to terminate every single layer with a defined optical thickness. One effect, which has to be mentioned in this context, is error compensation. Usually, this is a positive effect of optical monitoring, which compensates e.g. for deviations in optical constants by modification of physical thickness. Furthermore, errors in already deposited layers are corrected by thickness adjustments of following layers. As a consequence, the result will be in most cases slightly worse than the target specification but better than without compensation effect. In case of chirped mirrors with additionally defined GDD targets, this compensation effects can be counterproductive, because variations or inaccuracies in dispersion have a large impact on the GDD and cannot be easily compensated by thickness modifications. Hence, the last layers of chirped mirrors, which normally represent an adjustment of the optical impedance, have an essential influence on the GDD performance of the mirrors and are often controlled by classical methods like quartz crystal monitoring or time counting. These methods are not very effective, because many coating iterations will be needed to find a coating parameter set for a satisfactory GDD result. To attain information about the developing GDD, especially of these last growing layers, an in situ Fourier-transform measurement system is proposed. The method is based on a Michelson interferometer with a broad band light source and a fast spectrometer. To our knowledge, the measurement of the GDD directly on the moving substrates during the coating process has been shown for the first time. With this additional information about the group delay dispersion, novel real time algorithms can be developed to react on deposition errors and to optimize the thicknesses of the last layers of chirped mirror systems.

\section{THEORETHICAL BASIS}

\section{Fourier-Transform Spectral Interferometry}

Fourier-Transform Spectral Interferometry (FTSI) is an alternative to the classical time domain analysis method that is used for interference analysis. The main advantage is a fast data acquisition of the measured wide spectral range, which is essential for online in situ measurements on moving substrates. Details of the measurement equipment are given in the

Optical Systems Design 2015: Advances in Optical Thin Films V, edited by Michel Lequime,

H. Angus Macleod, Detlev Ristau, Proc. of SPIE Vol. 9627, 96271S - (c) 2015 SPIE

CCC code: $0277-786 \mathrm{X} / 15 / \$ 18 \cdot$ doi: $10.1117 / 12.2191215$

Proc. of SPIE Vol. 9627 96271S-1 
next section. In this section, the determination of the differences in phase between two light pulses by FTSI is described [1]. If $E_{R}(t)$ and $E_{S}(t)$ represent the time dependence of two electrical fields and $E_{R}(\omega)$ and $E_{S}(\omega)$ their Fourier transforms, the phase difference $\Delta \varphi$ to be measured is given by

$$
\Delta \varphi=\arg \left[E_{S}(\omega)\right]-\arg \left[E_{R}(\omega)\right]
$$

The resulting frequency spectrum of the interferometer, which is analyzed by a spectrometer, is given by

$$
\begin{gathered}
S(\omega)=\left|E_{R}(\omega)\right|^{2}+\left|E_{S}(\omega)\right|^{2}+2 \operatorname{Re}\left\{E_{R}{ }^{*}(\omega) E_{S}(\omega)\right\} \\
=\left|E_{R}(\omega)\right|^{2}+\left|E_{S}(\omega)\right|^{2}+E_{R}{ }^{*}(\omega) E_{S}(\omega) \times \exp [i(\omega \tau+\Delta \varphi)]+c . c .
\end{gathered}
$$

where c.c. stands for the complex conjugated of the previous term and $\tau$ for the relative time delay between the two interferometer arms. The last two terms represent the interference part and depend strongly on the phase difference of the two electrical fields. To extract the phase information, firstly an inverse Fourier transformation of Equation (2) into the time domain is applied:

$$
F T^{-1} S(\omega)=E_{R}^{*}(-T) \otimes E_{S}(T)+E_{S}^{*}(-T) \otimes E_{R}(T)+S(T-\tau)+S(-T-\tau)^{*}
$$

The first two terms are autocorrelation functions of the individual reflection signals of the reference mirror and the sample substrate. They are centered at $T=0$. In contrast to this, the terms $S(T-\tau)$ and $S(-T-\tau)^{*}$ are centered at $T=\tau$ and $T=-\tau$, respectively [2]. Hence, the values of $\tau$ should be large enough for the required separation of the signal components [3] [4] [5]. This evaluation can be done by convolution with a separation function, e.g. a super-Gauss window function

$$
w(T)=\exp \left[-(T / \sigma)^{2 \lambda}\right] .
$$

The parameter $\sigma$ denotes the half-width of the function and $\lambda$ the super-Gauss order [2] [6]. In a next step, the result is shifted by the time delay $-\tau$. Finally, a transformation back into the frequency domain

$$
s(\omega)=F T\left\{\left[F T^{-1} S(\omega)\right] \times \exp \left[-((T+\tau) / \sigma)^{2 \lambda}\right]\right\}
$$

results directly in the filtered signal $s(\omega)$ with phase difference $\Delta \varphi(\omega)=\arg [s(\omega)]$ [7].

If the influence of the reference mirror on phase is independent of frequency (see Paragraph 3), the group delay $G D(\omega)$ of the phase difference $\Delta \varphi(\omega)$ is equal to the group delay of the phase $\varphi_{S}(\omega)$ of the sample under inspection:

$$
G D(\omega)=-\frac{\partial}{\partial \omega} \arg [s(\omega)]=-\frac{\partial(\Delta \varphi)}{\partial \omega}=-\frac{\partial\left(\varphi_{S}\right)}{\partial \omega} .
$$

The group delay dispersion (GDD) can be calculated by an additional derivation of the GD with respect to the frequency $\omega[6][8]$.

$$
G D D(\omega)=-\frac{\partial(G D)}{\partial \omega}=\frac{\partial^{2}(\Delta \varphi)}{\partial \omega^{2}}
$$

\section{Signal requirements and Fourier approximations}

Signals that should be analyzed by Fourier transformations have to fulfill some essential requirements. If fast algorithms like FFT are used, the data points have to be evenly spaced. This can be implemented in a simple way by suitable sampling approaches. The second aspect, that has to be considered in case of Fourier transformations, is connected to the periodicity and differentiability of the functions. In practice, measured signals result in non-periodic jump discontinuities at the start and end of the measurement range. As a consequence, the Fourier series produce large artifacts in form of oscillations (Figure 4 and Figure 5). To overcome this issue, a common Fourier approximation is to multiply the signal with a periodic window function so that the product and its derivatives become periodic with respect to noise and computational accuracy [9] [10]. However, this approach influences the performance of the measurement system. On the one hand, the signal-to-noise ratio decreases especially at start and end of the measurement range. On the other hand, also the window function method produces artifacts. Therefore, the windowing has to be a compromise between enforcing the periodicity and optimizing the shape of the calculation result in dependency of the window function as well as the given data set [11]. Finally, the approximation is very sensitive to the noise of the signal and the window function parameters connected to the separation of interference terms. 
In case of the aimed in situ measurements on moving substrates, the effects mentioned above would result in strong oscillations of the GD and GDD curves. Hence, the Short Time Fourier Transformation (STFT) was chosen as an alternative and more suitable Fourier approximation method. The STFT is a well-known approach for wavelet analysis in many areas of signal processing [12] and can be also used to determine the GD. In the given case, the main advantages compared to other wavelet analysis methods are a reduced computational time, an effective reduction of transformation artifacts, and a lower influence of noise due to the overlapping of several small range Fourier transformations [13]. In particular, the usage of a window function for the measured signal $S(\omega)$ before applying the Fourier transformation into the time domain (Equation (3)) is not necessary.

\section{GD determination by Short Time Fourier Transformation}

The Short Time Fourier Transformation $\operatorname{STFT}_{h}(T, \omega)$ of a signal $s(T)$ at time $\mathrm{T}$ and frequency $\omega$ is a linear timefrequency representation defined as

$$
\operatorname{STFT}_{h}(T, \omega)=\int_{-\infty}^{\infty} h\left[T-t^{\prime}\right] s\left[t^{\prime}\right] e^{-i \omega t \prime} d t^{\prime}
$$

With substitution of $t^{\prime}=t+T$ the equation can be written as

$$
\begin{aligned}
\operatorname{STFT}_{h}(T, \omega) & =\int_{-\infty}^{\infty} h[-t] s[t+T] e^{-i \omega[t+T]} d t \\
& =e^{-i \omega T} \int_{-\infty}^{\infty} h[-t] s[t+T] e^{-i \omega t} d t \\
& =e^{-i \omega T} X(T, \omega)
\end{aligned}
$$

Where $h[-t]$ is a real-valued window function with finite length centered around zero, e.g. a Gauss window. However, in the following application example (see Paragraph 4) a flat-top window function was used because of its specific advantages in amplitude accuracy. Although the Gauss window produces fewer artifacts in the side bands and leads to better results at the edges of the spectral range, it disturbs the calculated GDD curves by small ripples (Figure 6).

The STFT in the form

$$
\begin{aligned}
X(T, \omega) & =\int_{-\infty}^{\infty} h[-t] s[T+t] e^{-i \omega t} d t \\
& =M(T, \omega) e^{i \varphi(T, \omega)},
\end{aligned}
$$

with the magnitude of the time frequency spectrum $M(T, \omega)$ and the phase $\varphi(T, \omega)$ is equivalent modulo the phase factor $e^{-i \omega T}$. This a more prevalent form in which the window is time-translated with the signal held to a fixed time. In general, the group delay can be derived from the phase $\varphi(T, \omega)$ (see Equation $(6)$ ). The STFT in the form $X(T, \omega)$ gives the spectral information of the signal within the window at its position in time. By sliding the window $h(T)$ to different positions, the time-varying spectral characteristic of the signal can be extracted [13] [14] [15] [16] [17] [18].

Based on Equation (10) the group delay can be directly determined by using the center-of-gravity method in direction of time [19]:

$$
G D(T, \omega)=\frac{\int_{-\infty}^{\infty} T X(T, \omega) d T}{\int_{-\infty}^{\infty} X(T, \omega) d T}
$$

The calculation of the GDD is given again by the derivative of the GD with respect to frequency $\omega$ (Equation (7)). 


\section{EXPERIMENTAL SETUP}

In this section the experimental setup and the measurement process are presented. Figure 1 shows a schematic illustration of the measurement system. It consists of a Michelson Interferometer with a broad band light source, a spectrometer with a fast and sensitive detector, and a polarization maintaining fiber coupler as major components. As reference a protected silver mirror was selected because of the relatively low phase influence. An advantage of the fiber coupled system compared to a free-beam setup is the enhanced flexibility. Based on the fiber approach nearly every process chamber can be equipped almost independent of installation conditions like flange positions, chamber dimensions or specific substrate holder constructions. However, to realize this flexibility polarization maintaining single mode fibers with a restricted wavelength range have to be employed. The usable wavelength range of the presented system is $800-880 \mathrm{~nm}$. This restriction results from the combination of the applied light source, the fiber coupler and the detector of the spectrometer. Nevertheless, the measurement system is modular, because one or more components can be substituted independently to enlarge or shift the wavelength range.

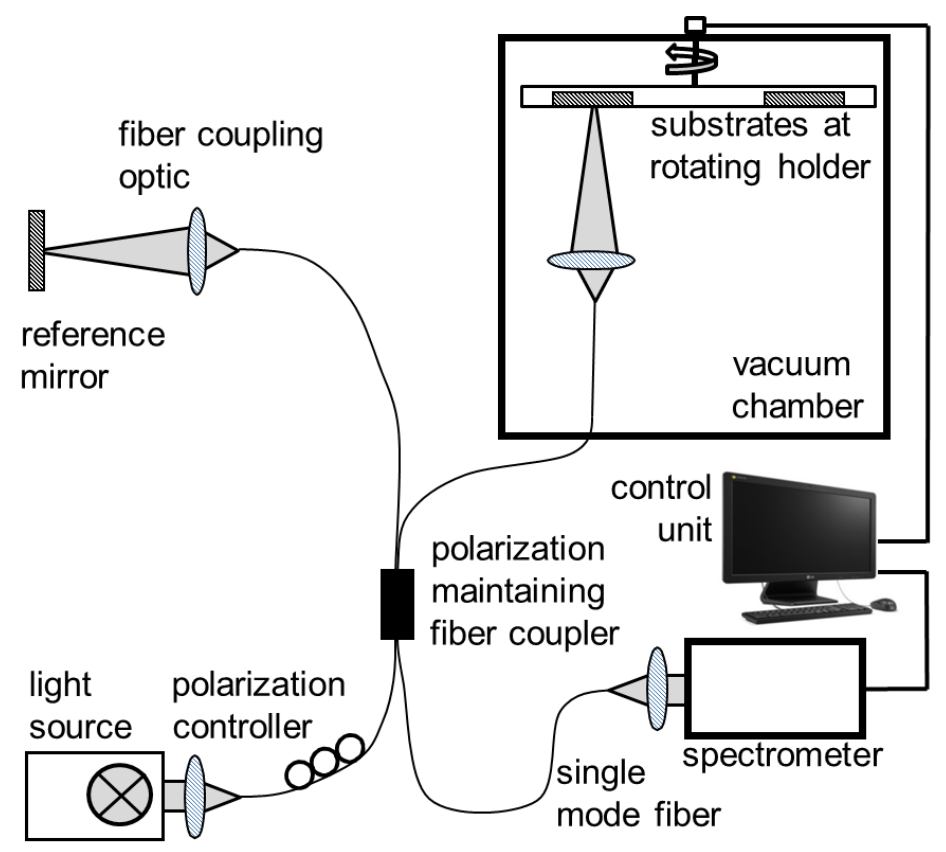

Figure 1: Experimental setup

\section{MEASUREMENT RESULTS}

\section{Data acquisition and transformation into time domain}

In this section, the measurement performance of the system is demonstrated by an example of a chirped mirror coating with $-600 \mathrm{fs}^{2}$ GDD in a wavelength range of 830 to $850 \mathrm{~nm}$. The developed measurement system determines the GDD directly on the rotating substrate holder. Depending on the rotation speed, the size of sample and the diameter of substrate holder, 20-60 measurements of the spectrum $S(\lambda)$ can be performed typically every rotation (see Figure 2). Following Equation (3), Figure 3 shows the real part of the Fourier transformed interferometric measurement $S(\omega)$ of the example coating (see Figure 2) and the super-Gauss separation function. The separated signal has to be shifted back to zero by the time delay $-\tau$ of around $500 \mathrm{fs}$. It should be mentioned that the width of the peaks (Figure 3 ) is increased by FFT artifacts. If the measurement is windowed by, for example, a Gauss function, the width will be decreased, but influenced by systematic artifacts of the window function. This will be discussed in more detail in the next section. 


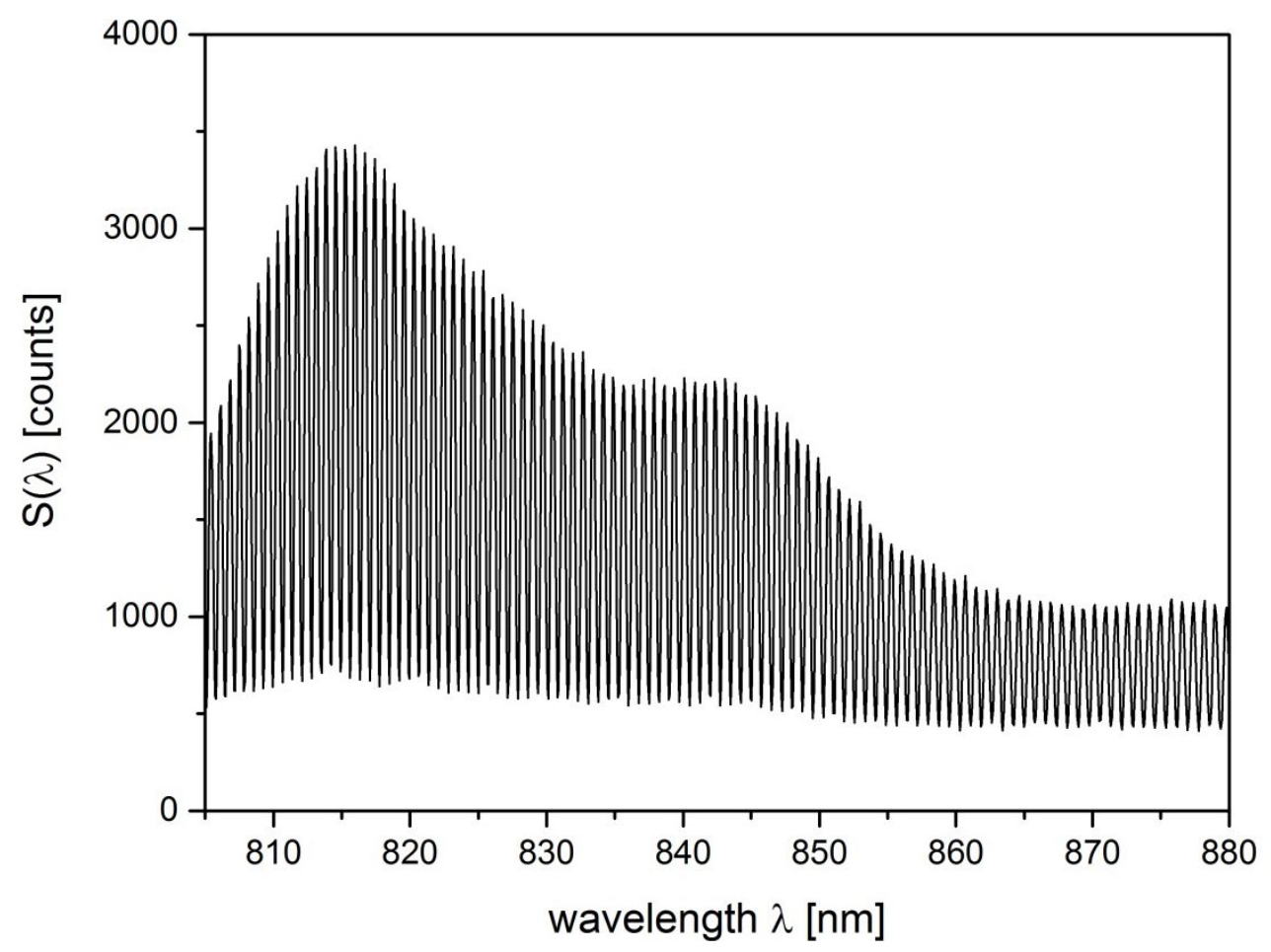

Figure 2: Non windowed in situ interferometric measurement of an example of a chirped mirror coating with $-600 \mathrm{fs}^{2}$ GDD

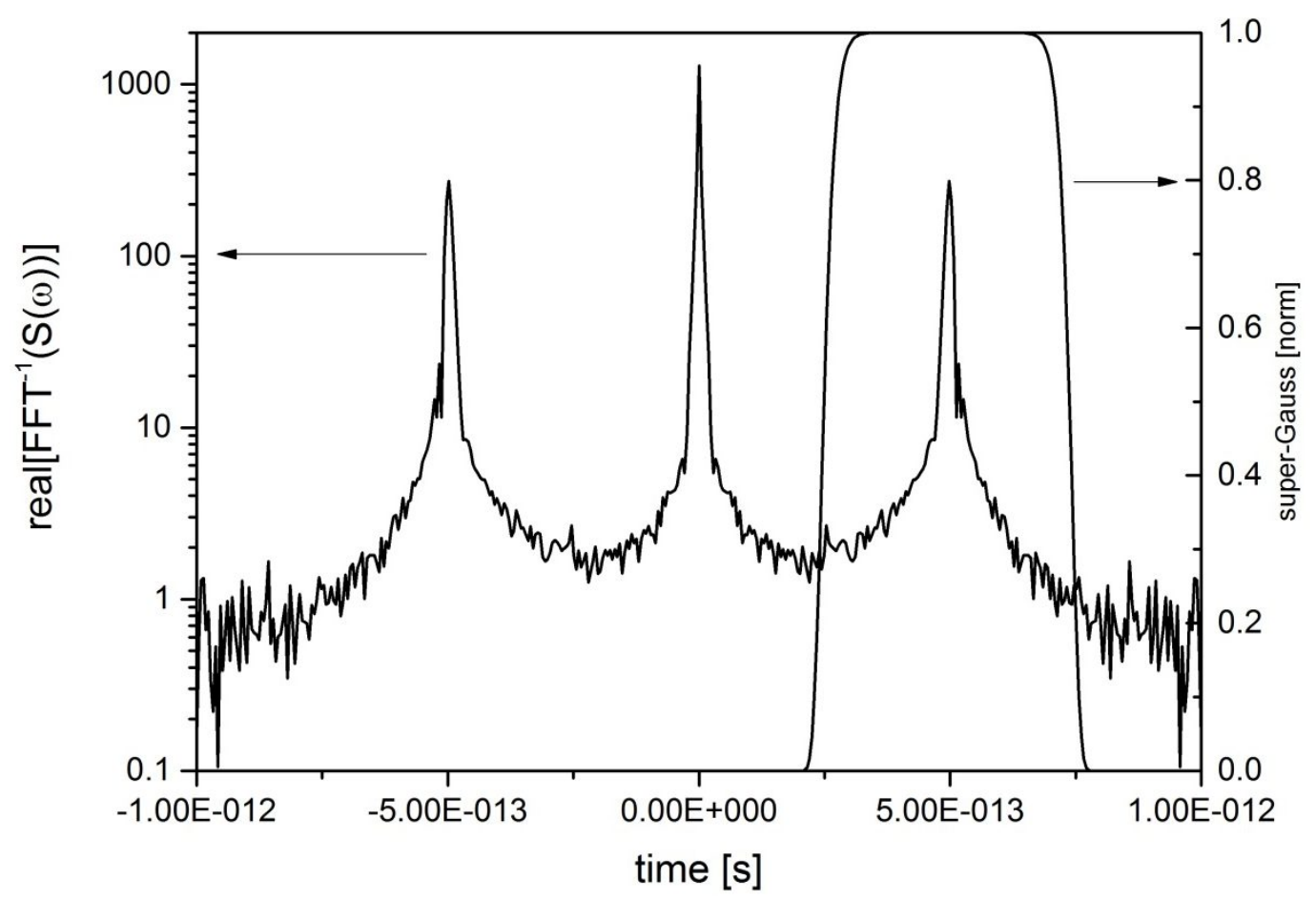

Figure 3: Real part of the Fourier transformed interferometric measurement $\boldsymbol{S}(\boldsymbol{\omega})$ and super-Gauss separation function 


\section{Comparison of different transformation procedures back into frequency domain}

In this section, results of different methods for the transformation back into the frequency domain are presented. Thereby, the advantages of the STFT method for the in situ GDD measurements, which are related to averaging and smoothing effects, are demonstrated. Figure 4 shows the consequences of an inappropriate interference peak separation. In this case, the applied super-Gauss function is too broad and causes in combination with the signal noise strong oscillations, although the measurement signal $S(\omega)$ was windowed with a Gauss function before FFT $^{-1}$ was applied. These disadvantageous oscillations distort the GDD if calculating with (Fig. 4) as well without windowing the measurement signal $S(\omega)$.

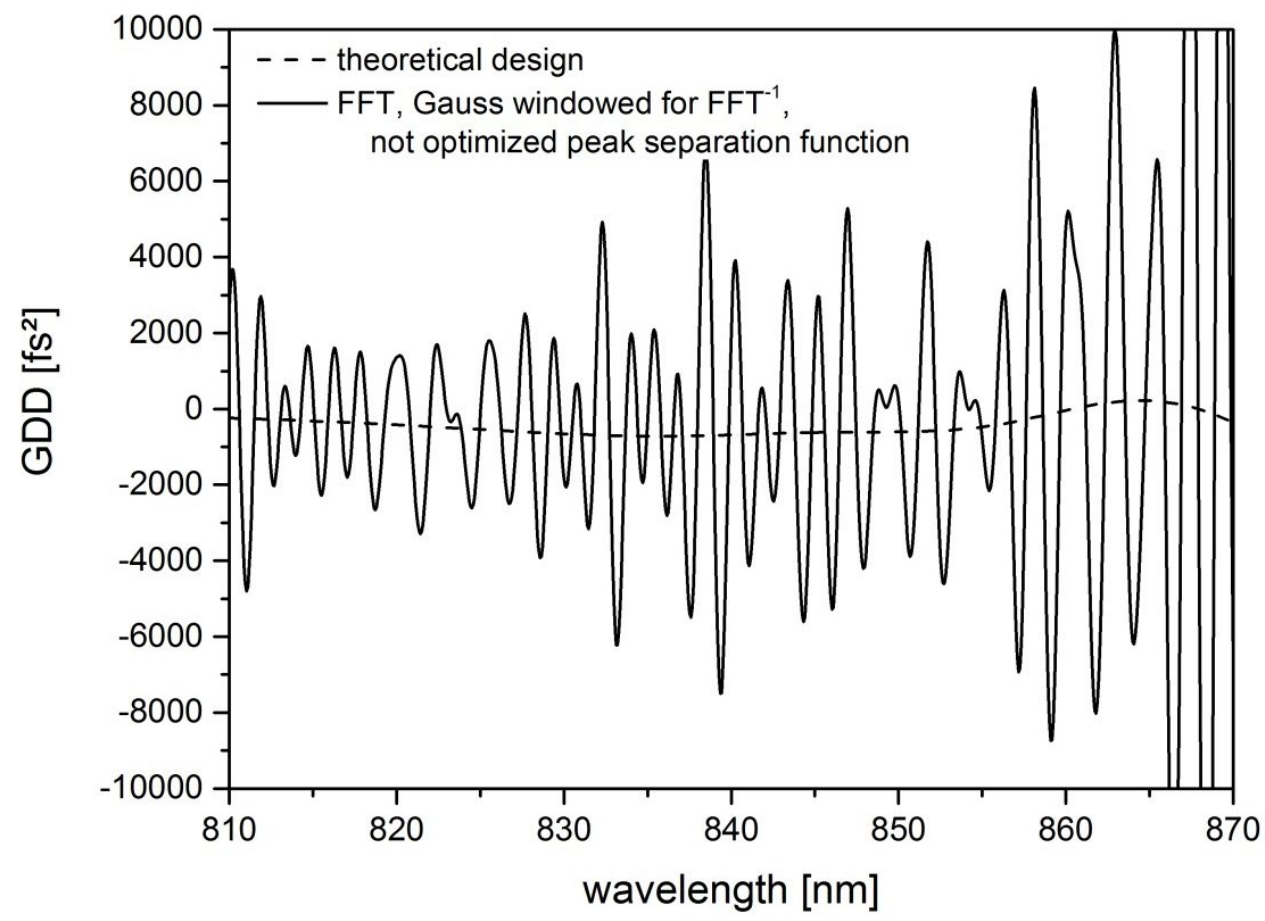

Figure 4: Influence of noise on calculated GDD, Gauss windowed FFT $^{-1}$, FFT with an exceedingly broad separation function for demonstration

In contrast, Figure 5 presents the results of an optimized peak selection for the FFT transformations. The light grey curve shows the case without a windowing of the measurement signal, and the dark grey curve represents the optimum result that is achievable with windowing and an optimized peak separation. However, both curves are worse than the STFT result (black) in comparison to the theoretical design curve (dashed). Additionally, there are transformation artifacts of the Gauss window function observable as systematic ripples for example between 830 and 840 nm wavelength. These artifacts can be observed also with applied Gauss window as moving window for STFT (Figure 6) in comparison with flat-top windowed STFT and the design curve. As mentioned before, a flat-top window is superior in this case and was used as default. Nevertheless, the usage of the STFT produces better and more stable results than the classical FFT even without an additional windowing before the transformation into time domain and without an optimization of the peak separation function. This is particularly important, because in case of the fast in situ measurement on moving substrates it is impossible to optimize the separation window parameters continuously. 


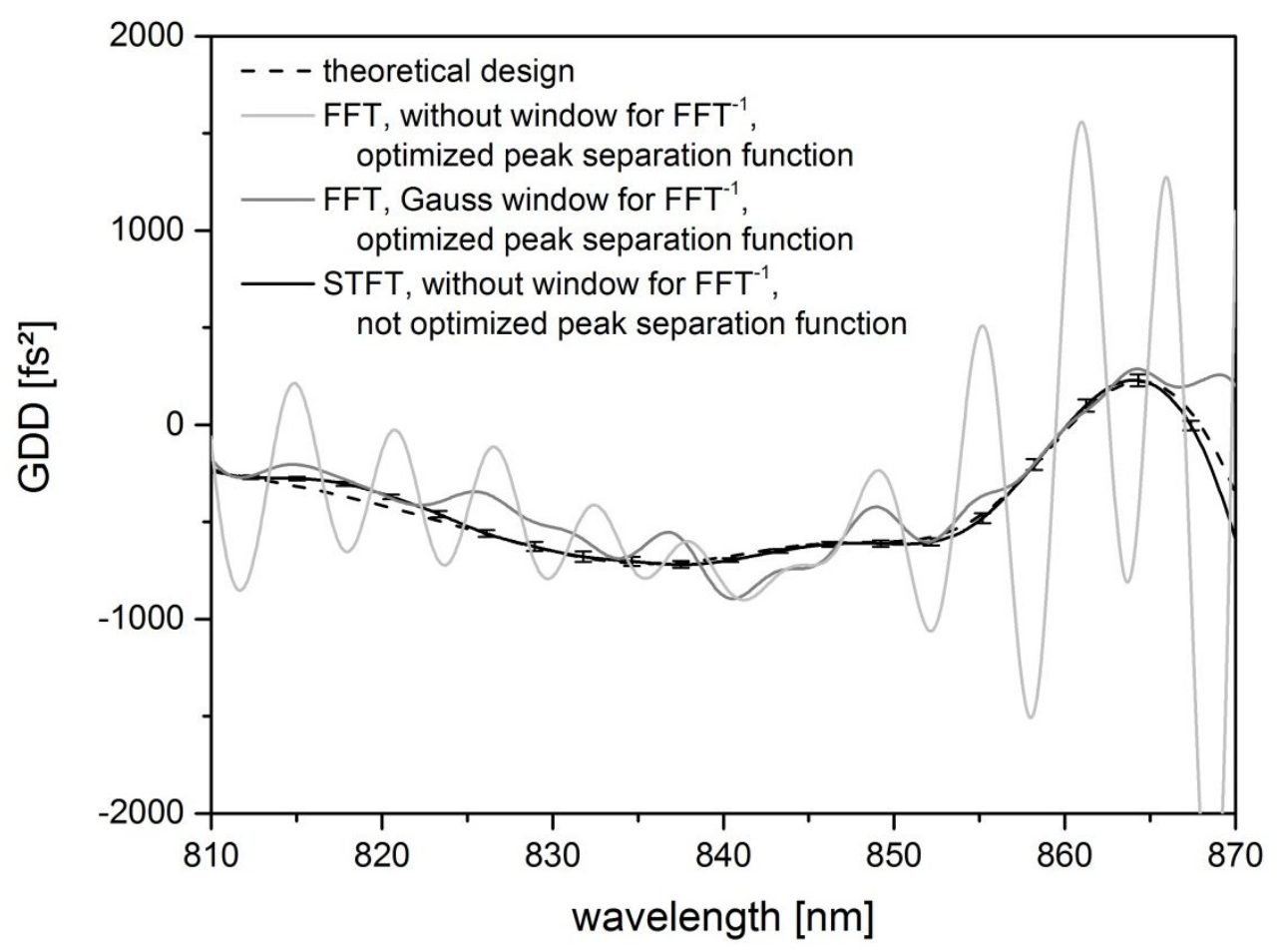

Figure 5: Calculated GDD influenced by separation function adjusting and windowing of the measurement signal for FFT ${ }^{-1}$

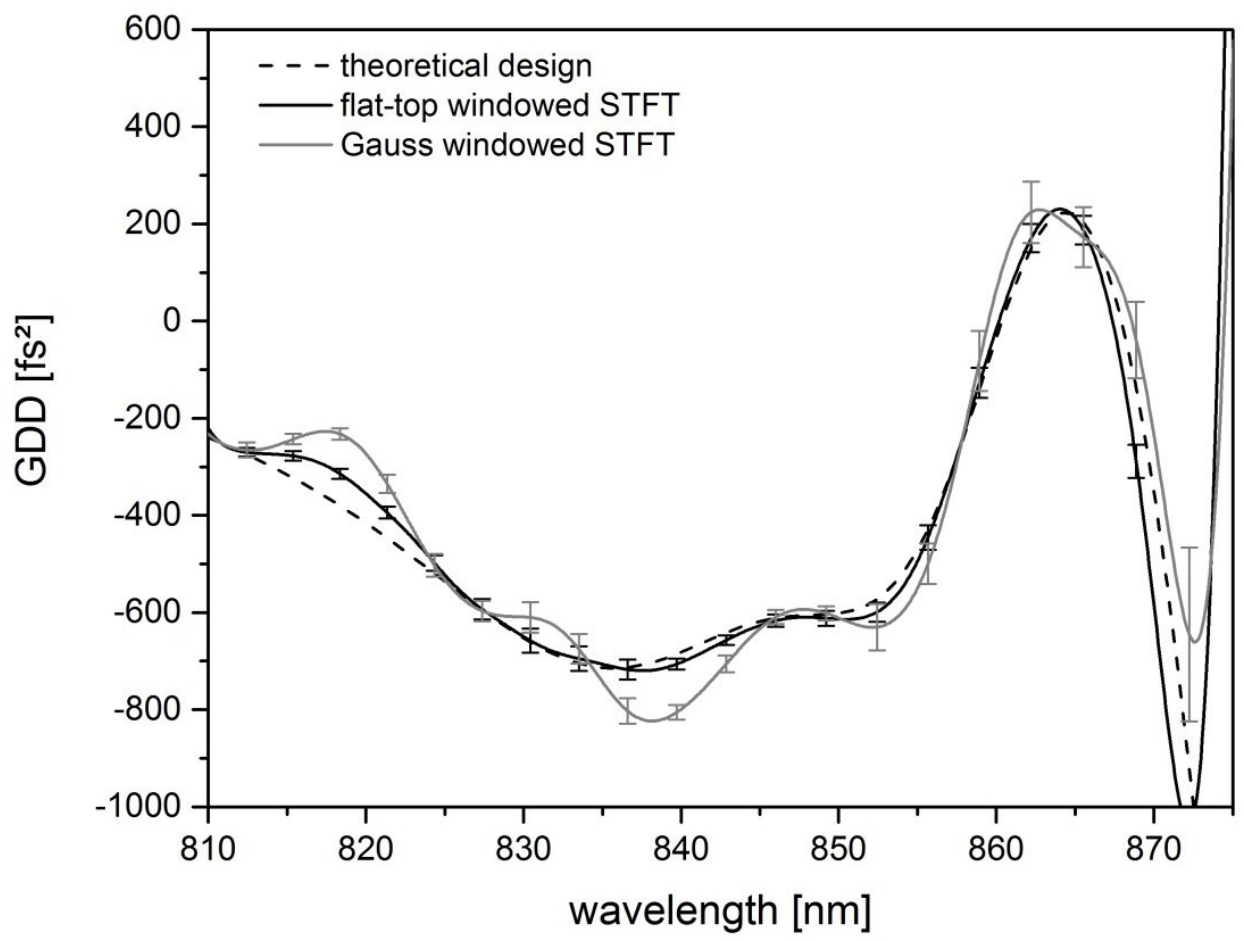

Figure 6: Comparison of STFT from time domain into frequency domain with Gauss and flat-top STFT window

Finally, Figure 7 shows the GDD result with usage of the STFT and a moving flat-top window in comparison to an ex situ measurement done with a Chromatis ${ }^{\mathrm{TM}}$ time domain GDD measurement system by KMLabs ${ }^{\mathrm{TM}}$. The measurement 
results differ slightly, which could be due to the different measurement spot sizes and measurement positions in combination with minor layer thickness inhomogeneities or substrate deformations caused by coating stress. In case of the Chromatis ${ }^{\mathrm{TM}}$ ex situ measurement system, the spot area is approximately one square centimeter and the measurement position slightly off-center. In contrast, the spot diameter of the in situ measurement system is in the range of $50 \mu \mathrm{m}$ and the measurement is done on a defined radius along the moving substrate. In the presented case about 40 from 60 measurements performed every revolution of the substrate holder are selected for data analysis. Measurements with, for example low interference contrast, are sorted out. The in situ measurement of Figure 7 is an average of 100 rotations and consists of about 4000 single measurements. The error bars of about $10 \mathrm{fs}^{2}$ in the range of $840 \mathrm{~nm}$ wavelength demonstrate the stability of the in situ measurement.

In addition, in Figure 7 two design curves which correspond to a layer thickness difference of only $2.2 \mathrm{~nm}$ in layer 53 of 55 are depicted (deviation of less than 1.5\%). The first curve matches very well with the in situ measurement and the other curve with the ex situ measurement of the Chromatis ${ }^{\mathrm{TM}}$ system. This illustrates how sensitive the GDD reacts on coating errors.

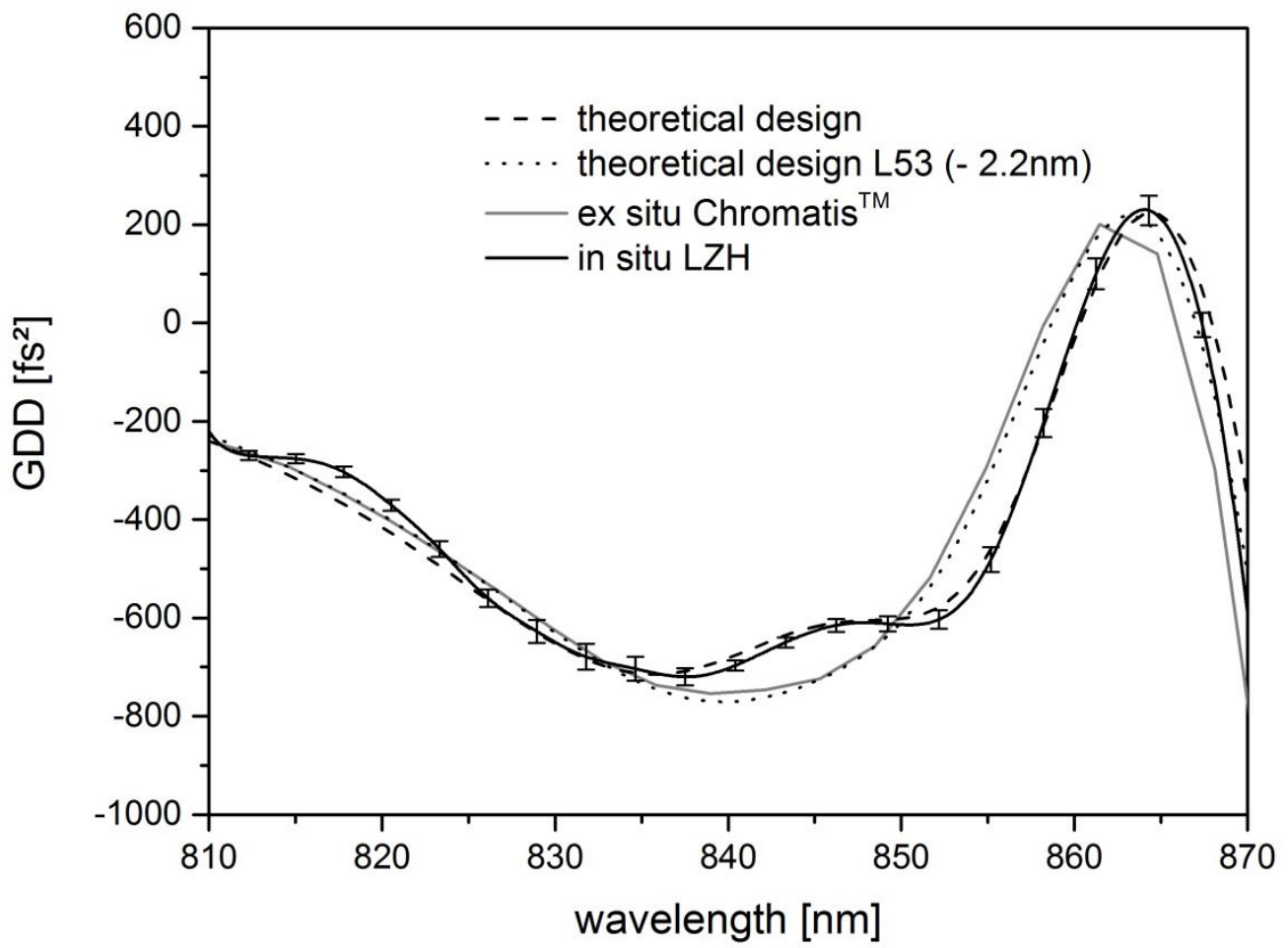

Figure 7: In situ GDD measurement in comparison to ex situ Chromatis ${ }^{\mathrm{TM}}$ measurement and theoretical design GDD

\section{CONCLUSIONS}

This study demonstrates a new group delay and group delay dispersion in situ measurement system for use in optical coating plants. With the presented fiber based FTSI system it is possible to measure the GD and GDD directly on the moving substrates during complex deposition processes. Different Fourier analysis methods were evaluated in respect to optimum stability. Finally, a conventional Fourier transformation into time domain without windowing functions and a subsequent STFT with a flat-top moving window back into frequency domain have been proven to be the most appropriate solution. This approach opens the way for a significantly enhanced chirped mirror production. Furthermore, based on the additional phase information new deposition control algorithms can be developed as the GDD is very sensitive to thickness variations and can be used for a more reliable differentiation between layer thickness and refractive index. 


\section{REFERENCES}

[1] Luo, Z., Liu, X., Zhang, S.; Shen, W., Xia, C. and Yin, Y., "Dispersive white-light spectral interferometer for optical properties measurement of optical thin films, "Chinese Optics Letters 8, pp. 99-101 (2010).

[2] Cagigas, M. A., Valle, P. J. and Cagigal, M. P. "Super-Gaussian apodization in ground based telescopes for high contrast coronagraph imaging," Optics Express 21(10), pp. 12744-12756 (2013).

[3] Dorrer, C. and Joffre, M., "Characterization of the spectral phase of ultrashort light pulses, “C. R. Acad. Sci. IV, pp. 1415-1426 (2001).

[4] Dorrer, C., Belabas, N., Likforman, J.-P. and Joffre, M., "Spectral resolution and sampling issues in Fouriertransform spectral interferometry,“ J. Opt. Soc. Am. B 17(10), pp. 1795-1802 (2000).

[5] Takeda, M., Ina, H. and Kobayashi, S., "Fourier-transform method of fringe-pattern analysis for computer-based topography and interferometry,“ J. Opt. Soc. Am. 72(1 ), pp. 156-160 (1982).

[6] Naganuma, K., Mogi, K., and Yamada, H., "Group-Delay Measurement Using the Fourier-Transform of an Interferometric Cross-Correlation Generated by White-Light,“ Opt. Lett. 15(7), pp. 393-295 (1990).

[7] Lepetit, L., Chériaux, G. and Joffre, M., "Linear techniques of phase measurement by femtosecond spectral interferometry for applications in spectroscopy,“J. Opt. Soc. Am. B 12(12), pp. 2467-2474 (1995).

[8] Fercher, A. F., Hitzenberger, C. K., Sticker, M., Zawadzki, R., Karamata, B. and Lasser, T., "Dispersion compensation for optical coherence tomography depth-scan signals by a numerical technique,“ Optics Communications 204, pp. 67-74 (2002).

[9] Rastogi, P., and Hack, E., [Phase Estimation in Optical Interferometry], Boca Raton: CRC Press (2015).

[10] Chen, P., "Gibbs Phenomenon Removal and Digital Filtering Directly through the Fast Fourier Transform, “ IEEE Trans. Signal Processing 49(2), pp. 444-448 (2001).

[11] Platte, R. B. and Gelb, A., "A Hybrid Fourier-Chebyshev Method for Partial Differential Equations, “ J. of Sc. Comp. 39(2), pp. 244-264 (2009).

[12] Durak, L. and Arikan, O., "Short-Time Fourier Transform: Two Fundamental Properties and an Optimal Implementation,“ IEEE Transactions on Signal Processing 51(5), pp. 1231-1242 (2003).

[13] Narasimhan, S. V. and Pavanalatha, S., "Estimation of evolutionary spectrum based on short time Fourier transform and modified group delay," Signal Processing 84, pp. 2139-2152 (2004).

[14] Fulop, S. A. and Fitz, K., "Algorithms for computing the time-corrected instantaneous frequency (reassigned) spectrogram, with applications,“ J. Acoust. Soc. Am. 119(1), pp. 360-371 (2006).

[15] Fulop, S. A. and Kelly, F., "Separation of components from impulses reassigned spectrograms, “ J. Acoust. Soc. Am. 121(3), pp. 1510-1518 (2007).

[16] Auger, F. and Flandrin, P. "Improving the Readability of Time-Frequency and Time-Scale Representations by the Reassignment Method,“ IEEE Transactions on Signal Proc. 43(5), pp. 1068-1089 (1995).

[17] Nelson, D. C., “' Cross-spectral methods for processing speech,” J. Acoust. Soc. Am., 110(5), pp. 2575-2592 (2001).

[18] Nelson, D. C., "Instantaneous Higher Order Phase Derivatives," Digital Signal Processing, 12(2-3), pp. 416-428 (2002).

[19] Flandrin, P., [Time-frequency/time-scale analysis], Academic Press (1998). 